\title{
EDS tomographic reconstruction regularized by total nuclear variation joined with HAADF-STEM tomography
}

\author{
Zhichao Zhong ${ }^{\mathrm{a}, *}$, Willem Jan Palenstijn ${ }^{\mathrm{a}}$, Jonas Adler ${ }^{\mathrm{c}, \mathrm{d}}$, K. Joost Batenburg ${ }^{\mathrm{a}, \mathrm{b}}$ \\ a Centrum Wiskunde \&' Informatica, Amsterdam, The Netherlands \\ ${ }^{\mathrm{b}}$ Mathematical Institute, Universiteit Leiden, Leiden, The Netherlands \\ ${ }^{\mathrm{c}}$ Department of Mathematics, KTH Royal Institute of Technology, Stockholm, Sweden \\ d Elekta, Stockholm, Sweden
}

\section{A R T I C L E I N F O}

\section{Article history:}

Received 7 February 2018

Revised 16 April 2018

Accepted 26 April 2018

Available online 5 May 2018

\begin{abstract}
A B S T R A C T
Energy-dispersive X-ray spectroscopy (EDS) tomography is an advanced technique to characterize compositional information for nanostructures in three dimensions (3D). However, the application is hindered by the poor image quality caused by the low signal-to-noise ratios and the limited number of tilts, which are fundamentally limited by the insufficient number of X-ray counts. In this paper, we explore how to make accurate EDS reconstructions from such data. We propose to augment EDS tomography by joining with it a more accurate high-angle annular dark-field STEM (HAADF-STEM) tomographic reconstruction, for which usually a larger number of tilt images are feasible. This augmentation is realized through total nuclear variation (TNV) regularization, which encourages the joint EDS and HAADF reconstructions to have not only sparse gradients but also common edges and parallel (or antiparallel) gradients. Our experiments show that reconstruction images are more accurate compared to the non-regularized and the total variation regularized reconstructions, even when the number of tilts is small or the X-ray counts are low.
\end{abstract}

(c) 2018 Elsevier B.V. All rights reserved.

\section{Introduction}

X-ray energy-dispersive spectroscopy (EDS) tomography is an electron tomography (ET) technique for 3D compositional characterization. It refers to making a tomographic reconstruction for the distribution of a specific chemical element from a tilt series of images called elemental maps. It is based on the linearity assumption that the image intensities, which correspond to X-ray counts, are proportional to the mass-thickness of the chemical element [1,2]. Unfortunately, the number of X-ray counts is often low due to small emission probabilities and small detection angles. Consequently, the signal-to-noise ratios (SNRs) are low and the number of tilts is limited, which leads to poorly reconstructed images. To obtain sufficient X-ray counts, a high electron dose is often applied by setting a large beam current or a long acquisition time. However, this is then limited by how much dose the sample can survive.

\footnotetext{
* Corresponding author.

E-mail addresses: zhong@cwi.nl (Z. Zhong), willem.jan.palenstijn@cwi.nl (W.J. Palenstijn), Jonas.Adler@elekta.com (J. Adler), joost.batenburg@cwi.nl (K.J. Baten-
} burg).
Using advanced reconstruction algorithms, it is possible to make an accurate reconstruction from a small number of tilts or from data with low SNRs. Total variation (TV) regularization algorithms are a widely-used method for reconstructing from a small number of tilts, which find a solution with sparse gradients to the ill-posed inverse problem [3-5]. In ET, it is adopted for reducing the missing wedge artifacts [6]. In EDS tomography with low-count data, it is used for the effect of suppressing noise and encouraging piecewise constant structures $[7,8]$. However, additional difficulties arise when TV regularization is applied to EDS tomography. The noise aggravates a significant issue of TV - the staircase effect that produces small flat regions separated by edges. In addition, as the TV regularization tends to preserve sharp discontinuities, the noisy edges become sharp and saw-like. When the number of tilts is small or the X-ray counts are low, these effects become even more severe as a result.

In this paper, while TV regularization encourages the information of sharp edges in the reconstructed image, we instead use total nuclear variation (TNV) regularization which also encourages common edges in multiple images [9-11]. Using TNV regularization, it is possible to augment the available data with extra information from another imaging modality. The other modality being exploited is high-angle annular dark-field (HAADF) STEM. Its image 
contrast depends on the atomic numbers of probed atoms. Therefore, the tomographic reconstruction based on HAADF-STEM shows a (weighted) sum of distributions for all chemical elements [12,13], which sometimes also contains sharp edges showing the variance of distributions. More importantly, due to a strongly reduced time constraint, more HAADF-STEM tilt images can be measured with higher SNRs in a relatively short time. Thus the HAADF-STEM reconstruction is usually more accurate and less noisy.

Our proposed method performs the EDS reconstruction together with a HAADF-STEM reconstruction with joint TNV regularization, from a tilt series of EDS maps and a tilt series of HAADF-STEM images. As an extension of TV imposed on multiple images, the TNV regularization also promotes the sparsity of gradients for each image. Hence, it has similar effects in terms of suppressing noise and preserving sharp discontinuities. Additionally, TNV regularization promotes joint reconstructions that have common edge locations and gradients in the parallel/antiparallel directions. The TNV regularization can penalize the staircase effects and saw-like edges in the EDS reconstruction for not having coinciding edges in the HAADF-STEM reconstruction image.

Note that the staircase effects can also be reduced using total generalized variation (TGV) regularization which incorporates smoothness information of the reconstructed image. However, the TGV regularization incorporates no additional information from extra data. Thus, saw-like edges may be still present in the TGV regularized reconstruction. It is also noteworthy that the proposed method can be seen as a bimodal tomography approach. In a previous paper [14], we have proposed another bimodal method which is named HAADF-EDS bimodal tomography (HEBT). That method incorporates a different prior, exploiting that the HAADF data should be a linear combination of the EDS data for all chemical elements in the specimen. Therefore, HEBT can only be used if EDS data for all chemical elements in this sample have been acquired. In contrast, the TNV-regularized method only uses the data for a single chemical element in addition to the HAADF-STEM data.

The rest of this paper consists of the following sections. Section 2 illustrates the notations for EDS tomography, HAADF-STEM tomography, TNV regularization as well as the TNV-regularized joint reconstruction method. Section 3 demonstrates simulation studies and an experimental study. Lastly, in Section 4 we discuss the experimental results and draw a conclusion.

\section{Method}

Consider a specimen located in a 3D volume space discretized into $N$ voxels. The reconstruction unknowns for a single given chemical element in the specimen are expressed as a vector $\mathbf{x}^{e} \in$ $\mathbb{R}^{N}$. The reconstruction $\mathbf{x}^{e}$ is referred to as the EDS reconstruction. The input data are a tilt series of elemental maps, which are expressed as a vector $\mathbf{p}^{e} \in \mathbb{R}^{M^{e}}$, where $M^{e}$ denotes the total number of pixels. The image intensities correspond to the characteristic Xray counts, which are proportional to the linear projection of the corresponding chemical element probed by the focused beam under thin film approximation. This linear relationship is modeled by the following system of equations:

$p_{i}^{e}=\sum_{j=1}^{N} w_{i j}^{e} x_{j}^{e}$,

for $\quad i=1, \cdots, M^{e}$ and $j=1, \cdots, N$. Each pixel position $i$ corresponds to a ray determined by the beam position and the tilt angle of the specimen. The weight factor $w_{i j}^{e}$ is determined by the area of the $j$ th voxel intersected by the $i$ th ray integral. The matrix $\mathbf{W}^{e} \in \mathbb{R}^{M^{e} \times N}$ is referred to as the EDS projection matrix.
The volume space for the HAADF-STEM reconstruction is defined as the same for joining the reconstruction with the EDS reconstruction. Similarly, the reconstruction unknowns for the same sample are expressed as a vector $\mathbf{x}^{h} \in \mathbb{R}^{N}$, with $h$ denoting HAADFSTEM. The tilt series of projection images are expressed as a vector $\mathbf{p}^{h} \in \mathbb{R}^{M^{h}}$, where $M^{h}$ is the total number of pixels for HAADF-STEM acquisition. Note that $M^{h}$ may be different from the number of pixels for EDS acquisition $\left(M^{e}\right)$. In particular, this means it is possible to record HAADF-STEM data for more tilts than for which EDS data are acquired. Following the linear integral model, the linear relationship between the tilt series $\mathbf{p}^{h}$ and reconstruction unknowns $\mathbf{x}^{h}$ is:

$p_{k}^{h}=\sum_{j=1}^{N} w_{k j}^{h} x_{j}^{h}$,

where $k=1, \cdots, M^{h}$ is the pixel index, $w_{k j}^{h}$ is the entry of the HAADF-STEM projection matrix $\mathbf{w}^{h} \in \mathbb{R}^{M^{h} \times N}$ determined by the area of voxel $j$ intersected by the ray integral $k$.

Reconstruction algorithms can be divided into two categories: analytical algorithms (e.g., filtered back-projection (FBP) [15]) and iterative algorithms. Here we focus on iterative algorithms for their capability of implementing regularization. Classically, the iterative algorithm minimizes a data cost function, based on the above linear systems, to find solutions for the inverse problems

$$
\begin{aligned}
& \mathbf{x}^{e *}=\underset{\mathbf{x}^{e}}{\operatorname{argmin}}\left\|\mathbf{p}^{e}-\mathbf{W}^{e} \mathbf{x}^{e}\right\|^{2}, \\
& \mathbf{x}^{h *}=\underset{\mathbf{x}^{h}}{\operatorname{argmin}}\left\|\mathbf{p}^{h}-\mathbf{W}^{h} \mathbf{x}^{h}\right\|^{2},
\end{aligned}
$$

for EDS and HAADF-STEM tomography respectively.

To regularize the reconstruction, a regularization term is added to the cost function, resulting in a new minimization problem. Taking TV-regularized EDS tomography as an example, the reconstruction is computed by solving the minimization problem of:

$\mathbf{x}^{e *}=\underset{\mathbf{x}^{e}}{\operatorname{argmin}}\left\|\mathbf{p}^{e}-\mathbf{W}^{e} \mathbf{x}^{e}\right\|^{2}+\lambda \operatorname{TV}\left(\mathbf{x}^{e}\right)$,

where the term $\operatorname{TV}\left(\mathbf{x}^{e}\right)$ gives the total variation of the reconstruction image, and $\lambda$ is the factor determining the strength of the TV regularization.

The TV term for an arbitrary 3D gray-scale image $\mathbf{u} \in \mathbb{R}^{N}$ is defined as:

$\operatorname{TV}(\mathbf{u})=\sum_{j}^{N}\left\|\nabla u_{j}\right\|$,

where

$\nabla u_{j}=\left(\begin{array}{c}\nabla^{x} u_{j} \\ \nabla^{y} u_{j} \\ \nabla^{z} u_{j}\end{array}\right)$

is a discrete approximation of the gradient for the $j$ th voxel. The operators $\nabla^{x}, \nabla^{y}$ and $\nabla^{z}$ approximate gradients in the $\mathrm{X}, \mathrm{Y}$ and $\mathrm{Z}$ directions respectively by taking the forward difference between voxels. The norm $\|\cdot\|$ is usually chosen as $l_{1}$-norm or $l_{2}$-norm. In this paper, we use the $l_{2}$-norm, for which the TV regularization is also called isotropic TV. Using $l_{1}$-norm TV tends to encourage horizontal and vertical edges, which is a drawback. In contrast, $l_{2}$-norm TV is rotationally invariant and thus is preferable in the application of EDS tomography [3,9].

Next, we describe the notation for TNV regularization. TNV is usually imposed on images with multiple channels such as RGB images. In this paper, we join an EDS reconstruction image and a HAADF-STEM reconstruction as the two channels for one image. Consider an arbitrary $L$-channel image $\mathbf{u}$. The image intensity on the $j$ th pixel can be expressed as $\mathbf{u}_{j}=\left(u_{j}^{(1)}, \ldots u_{j}^{(2)} \ldots, u_{j}^{(L)}\right)^{T}$. 


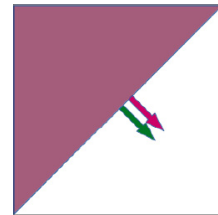

(a) Parallel gradients (b)

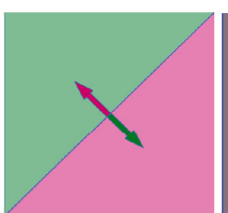

(b)

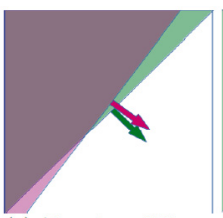

(c) Al

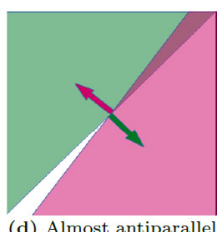

(d) Almo
Fig. 1. Illustration of TNV for two-channel images and gradients.

The TNV of $\mathbf{u}$ is then defined as the nuclear norm of the Jacobian matrix:

$\operatorname{TNV}(\mathbf{u})=\sum_{j}\left\|(\mathbf{J}(\mathbf{u}))_{j}\right\|_{\star}$,

where the $(\mathbf{J}(\mathbf{u}))_{j}$ is the Jacobian matrix defined by:

$(\mathbf{J}(\mathbf{u}))_{j}=\left(\begin{array}{c}\nabla^{x} u_{j}^{(1)}, \nabla^{y} u_{j}^{(1)}, \nabla^{z} u_{j}^{(1)} \\ \vdots \\ \nabla^{x} u_{j}^{(L)}, \nabla^{y} u_{j}^{(L)}, \nabla^{z} u_{j}^{(L)}\end{array}\right)$,

and the nuclear norm $\|\cdot\|_{\star}$ is defined as the $l_{1}$-norm of the Jacobian matrix' singular values. Note that the $l_{1}$-norm here does not encourage horizontal and vertical edges. For a one-channel image, the TNV is reduced to the isotropic TV.

TNV regularization introduces the following effects by encouraging the rank-sparsity in the Jacobian matrix. First of all, TNV regularization leads to the similar effects as TV regularization in terms of promoting the sparsity of image gradients, preserving sharp discontinuities and suppressing noise. The TNV is also rotationally invariant like the isotropic TV. Second, the TNV regularization gives preference to the images that have common edge locations and parallel or antiparallel gradient vectors, while it does not introduce false features between channels [9].

Fig. 1 illustrates examples of TNV computed for two-channel images with gradients pointing in parallel, antiparallel, almost parallel and almost antiparallel directions. For the parallel and antiparallel examples, one of the two singular values of the Jacobian matrix will be zero, while for the other two examples, the two singular values will be non-zero and unique. Suppose the norms of all gradients are equal to 1 , the TNV values for the pixels where two edges cross for (a), (b), (c) and (d) are 2, 2, 2.14 and 2.14 respectively. Therefore, minimizing TNV gives preference to the parallel or antiparallel gradients.

In our application of jointly regularizing the EDS and HAADFSTEM reconstructions, the data fit terms for EDS and HAADF-STEM

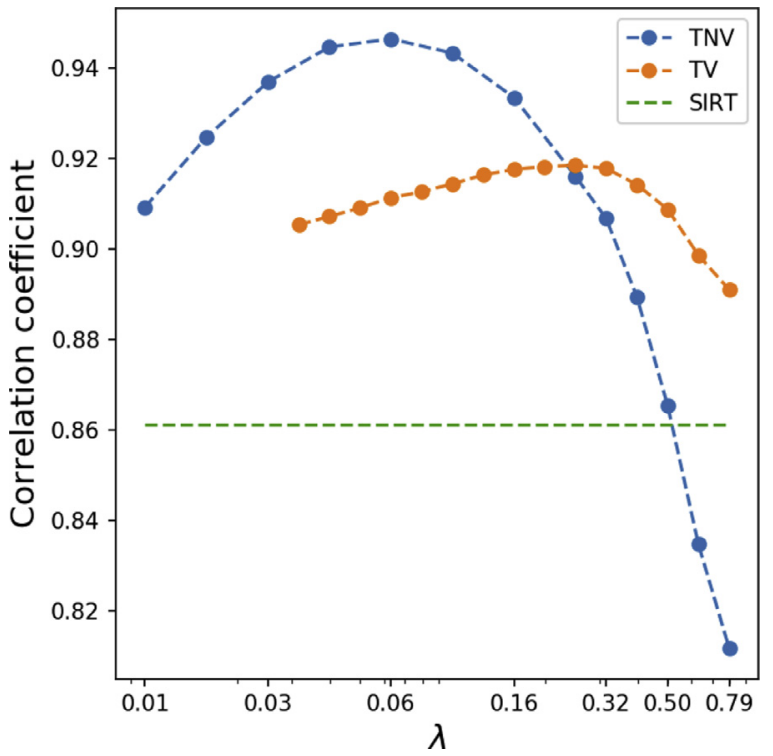

Fig. 3. Correlation coefficients w.r.t. regularization parameter values $(\lambda)$ in the coreshell phantom simulation study computed for different reconstruction methods. The SIRT reconstruction is performed for a fixed relaxation parameter set to 1 [18].

are minimized together with a TNV term:

$$
\begin{aligned}
& \mathbf{x}^{e *}, \mathbf{x}^{h *}=\underset{\mathbf{x}^{e}, \mathbf{x}^{h}}{\operatorname{argmin}}\left\|\mathbf{p}^{e}-\mathbf{W}^{e} \mathbf{x}^{e}\right\|^{2} \\
&+\left\|\mathbf{p}^{h}-\mathbf{W}^{h} \mathbf{x}^{h}\right\|^{2}+\lambda \operatorname{TNV}\left(\mathbf{x}^{e}, \mathbf{x}^{h}\right)
\end{aligned}
$$

where $\operatorname{TNV}\left(\mathbf{x}^{e}, \mathbf{x}^{h}\right)$ represents the TNV for a two-channel image consisting of $\mathbf{x}^{e}$ and $\mathbf{x}^{h}$, which means $L=2$ and $\mathbf{u}_{j}=\left(x_{j}^{e}, x_{j}^{h}\right)^{T}$ for Eq. (9). $\lambda$ is the regularization parameter determining the strength of TNV regularization. In practice, the value of $\lambda$ should be chosen carefully to obtain a desired reconstruction result. A too large value may lead to an over-regularized image with blurred edges, while a too small value may lead to insufficient regularization effects.

In practice, we have to consider the magnitude of these two types of input data. The magnitudes of EDS elemental maps and HAADF-STEM images can be tremendously different. Intensities of the EDS elemental map, which correspond to the X-ray counts, are usually of the order of magnitude 1 or 2 , while intensities of the HAADF-STEM image usually have different magnitudes. This difference of magnitude may cause a biased TNV term. To avoid this, we scale the image intensities of both types of images to a range from 0 to 1 before the reconstruction step. Afterwards the reconstructed image can be re-scaled by the same value so that quantitative characterization is still feasible.

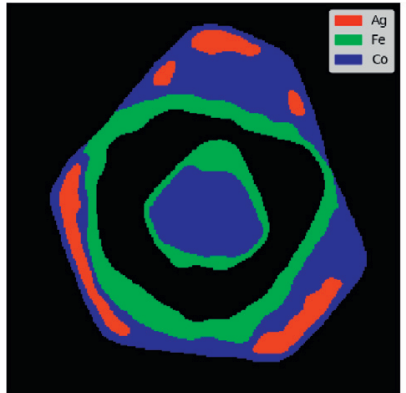

(a) Distributions of the chemical elements.

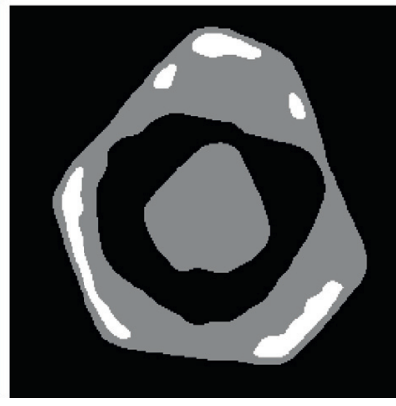

(b) HAADF-STEM phantom

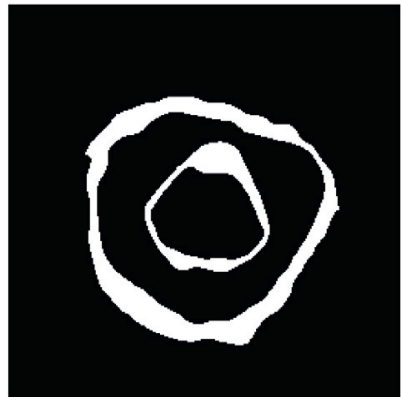

(c) EDS phantom for $\mathrm{Fe}$

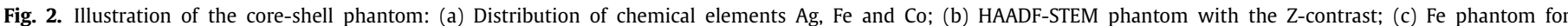

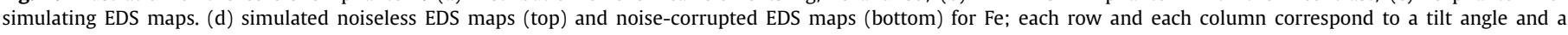
beam position respectively. 

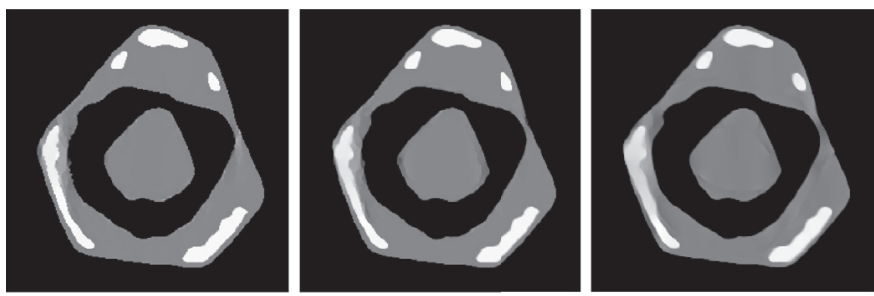

$\lambda=0.01$

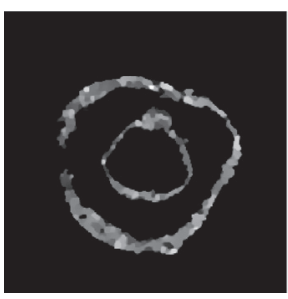

$\lambda=0.06$

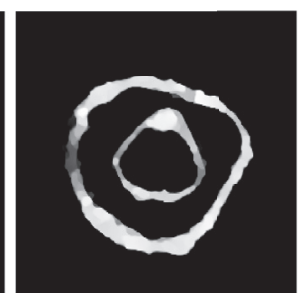

(e) $\operatorname{EDS}, \lambda=0.06$ $\lambda=0.25$

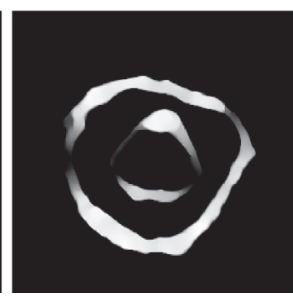

(f) $\mathrm{EDS}, \lambda=0.25$

Fig. 4. TNV-regularized joint reconstructions for the core-shell nanoparticle phantom simulation: (a)-(c) HAADF-STEM reconstructions corresponding to different values of regularization parameter $\lambda$; (d)-(f) EDS reconstructions corresponding to different values of regularization parameter $\lambda$.

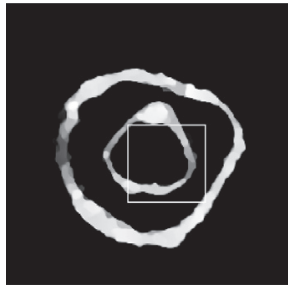

(a) $\mathrm{TNV}, \lambda=0.06$

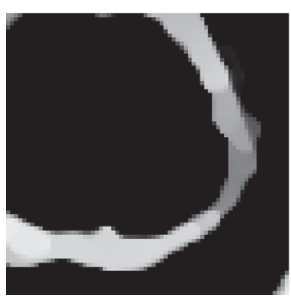

(d) Zoom-in to (a)

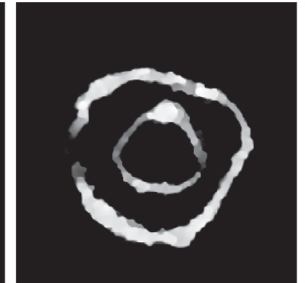

(b) $\mathrm{TV}, \lambda=0.25$

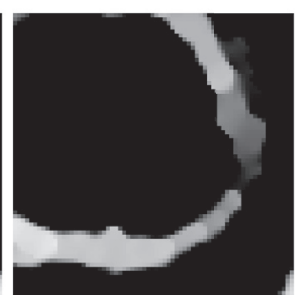

(e) Zoom-in to (b)

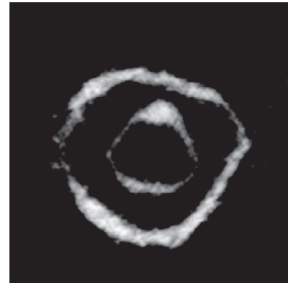

(c) SIRT

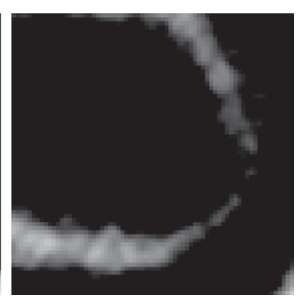

(f) Zoom-in to (c)
Fig. 5. Reconstructions for the core-shell nanoparticle phantom simulation: (a)-(c) EDS reconstructions using TNV, TV and SIRT corresponding to the optimal $\lambda$ values; (d)-(f) Regions of interest (RIO) in (a), (b) and (c) respectively. The white box in (a) indicates where the RIO is, which is the same for (b) and (c).

\section{Experiments}

In this section, we first investigate the proposed method based on a phantom simulation dataset and a multi-slice simulation dataset, for which ground-truth images are available. After that, we apply the method to a real experimental dataset.

For the simulation datasets, noiseless EDS maps are first computed. Based on the assumption that the noise is Poissondistributed with expected values given by the noiseless maps, we generate maps with Poisson noise similarly to the noise generation in [16] as follows: for each pixel on each map, a random number is generated for a Poisson distribution taking the corresponding noiseless image intensity as the expected value. This is then taken as the noise-corrupted image intensity. For a Poisson distribution the SNR is given by the square root of the expected value. The magnitude (and therefore the noise level) of the EDS maps could be calculated from assumed values of probe current, dwell time, fluorescence yield, solid angle and detection efficiency [16,17]. In this paper, for simplicity, we set the magnitudes to levels similar to real experimental data (up to $\sim 100$ counts).

For comparison, reconstructions are also made for a commonly used non-regularized reconstruction method - the simultaneous iterative reconstruction technique (SIRT) [18], and the TV regularization method defined by Eq. (5). We use the SIRT implementation in the ASTRA Toolbox [19], and use the Douglas-Rachford primaldual splitting algorithm implemented on the Operator Discretization Library (ODL) [20] in Python to compute the regularized reconstructions. SIRT is computed for 50 iterations to avoid overfitting to noise, while the regularized reconstruction is computed until convergence. We only perform 2D reconstructions for slices, but the results can be generalized to the third dimension for the same effects. For conciseness, we refer to the EDS reconstructed image jointly made using the TNV regularization as the TNV reconstruction, the reconstructed image regularized by TV as the TV reconstruction and the reconstructed image using SIRT as the SIRT reconstruction.

We use the linear correlation coefficient to measure image quality, which determines the extent to which the reconstructed image $\mathbf{u}$ is linearly related to the ground-truth image $\mathbf{g}$. The correlation coefficient $r$ is calculated as:

$r=\frac{\sum_{i}\left(u_{i}-\bar{u}\right)\left(g_{i}-\bar{g}\right)}{\sqrt{\sum_{i}\left(u_{i}-\bar{u}\right)^{2} \sum_{i}\left(g_{i}-\bar{g}\right)^{2}}}$,

where $\bar{u}$ and $\bar{g}$ are the mean values of $\mathbf{u}$ and $\mathbf{g}$ respectively.

We also compute segmentation errors which can indicate the accuracy of simple quantitative characterization. The segmentation error is calculated for a binary image segmented from a reconstructed image. It is defined as the proportion of incorrectly segmented pixels to the total number of non-zero pixels in the ground-truth binary segmentation. For the binary image $\mathbf{s}$ and the ground-truth binary image $\mathbf{t}$, the segmentation error $e$ is:

$e=\frac{\sum_{i}\left|s_{i}-t_{i}\right|}{\sum_{i} t_{i}}$,

for $s_{i} \in\{0,1\}$ and $t_{i} \in\{0,1\}$. The reconstruction image is segmented by thresholding the image intensities. To find the optimal thresholds, we calculate the segmentation errors for a set of thresholds between the minimal and maximal image intensities of the reconstructed image. Then the one corresponding to the minimal segmentation error is chosen as the optimal threshold, and the corresponding segmentation is adopted as the optimal segmentation.

\subsection{Phantom simulation}

\subsubsection{Data simulation and preparation}

The 2D phantoms are created to simulate the single slice of a core-shell particle composed of three chemical elements: Ag, Fe and Co. Fig. 2(a) shows the distributions for these elements. Fe and Co have very similar atomic numbers and consequently show the same contrast in the HAADF-STEM reconstruction image. Au, which is embedded in the outer shell, has a relatively large atomic number and a strong contrast in the HAADF-STEM image. A purpose of this phantom study is to investigate whether the strong $\mathrm{Ag}$ features in the HAADF-STEM reconstruction will introduce false features to the EDS reconstruction.

There are two phantoms in this example: a HAADF-STEM phantom and an EDS phantom for Fe, which are respectively shown in Fig. 2(b) and Fig. 2(c). We simulated the HAADF-STEM projection data using the ASTRA Toolbox for every $1^{\circ}$ from $20^{\circ}$ to $160^{\circ}$ for a limited angular range to create a missing wedge. After that, we added Gaussian noise into the data. In addition, we used the ASTRA Toolbox to simulate projection data for the Fe phantom for a 


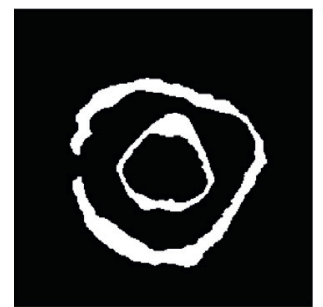

(a) $\mathrm{TNV}$

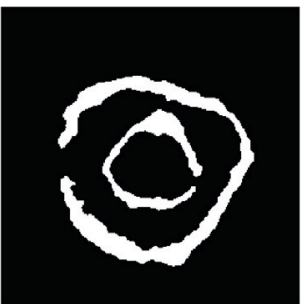

(b) TV

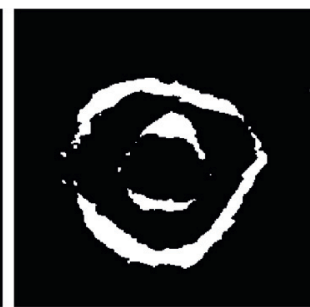

(c) SIRT

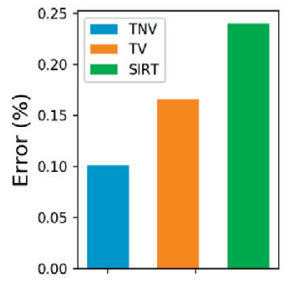

(d) Segmentation errors

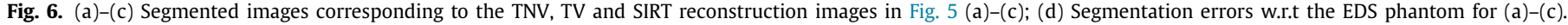

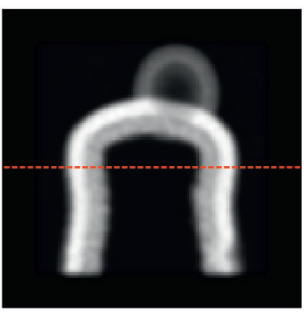

(a) HAADF-STEM image at (b) $2^{\circ}$

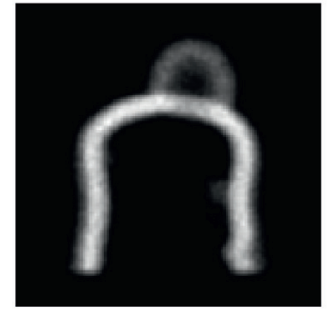

(c) EDS elemental map at $2^{\circ}$

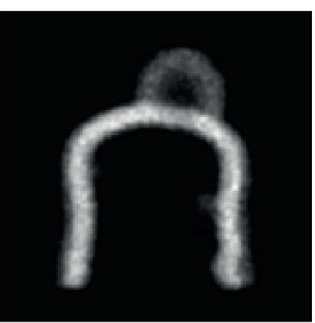

(e) EDS elemental map at $2^{\circ}$, $1 / 3$ photon counts

Fig. 7. Examples of simulated tilt images for the multislice simulation. The elemental maps for Ta shown here are after Gaussian smoothing. The red dashed lines indicate the position of the reconstructed slice. The colorbars indicate the image intensities. The tilt axis is vertical.

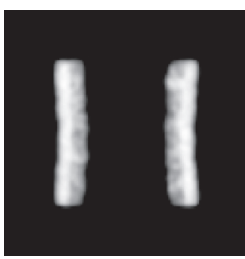

(a) HAADF reconstruction

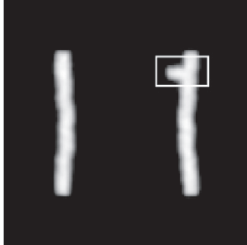

(b) EDS reconstruction for $\mathrm{Ta}$

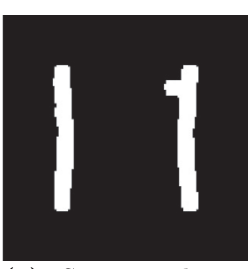

(c) Segmented image for (b)
Fig. 8. Ground-truth for the multi-slice simulation. The white box in (b) indicates where the 'tip' feature is.
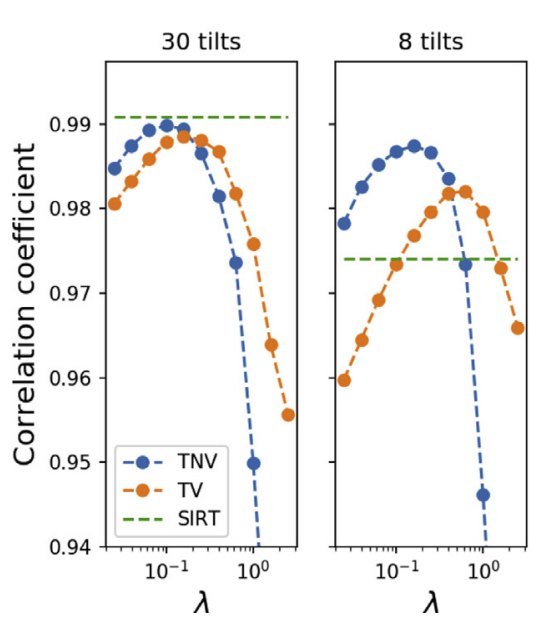

$1 / 3$ photon counts

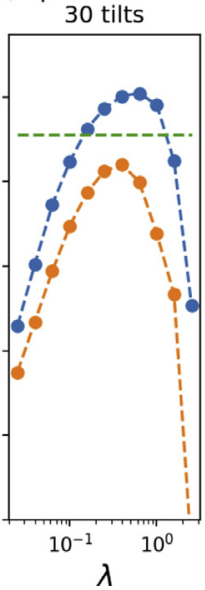

Fig. 9. Correlation coefficients w.r.t. regularization parameter $\lambda$ for different numbers of tilts and magnitudes of image intensities in the multi-slice simulation study. The SIRT reconstructions are performed for a fixed relaxation parameter set to 1 .

small number of tilts within the same angular range, which are for every $14^{\circ}$ from $20^{\circ}$ to $160^{\circ}$ (Fig. $2(\mathrm{~d})$ ). The intensities of the noiseless maps were scaled and then used to generate the EDS maps corrupted by Poisson noise. In this case, the average image intensity on the non-background pixels is 43.1. In the last step, the maps were filtered using a Gaussian filter $(\sigma=0.8$ pixel $)$ as a denoising process.

\subsubsection{Reconstruction results}

First of all, we performed TNV-regularized reconstructions for a set of regularization parameter $\lambda$ values to inspect the impact of this factor. We then plotted the correlation coefficients for these reconstructions in Fig. 3. Similarly, we performed TV reconstructions for various $\lambda$ values and a SIRT reconstruction. The correlation coefficients are also plotted in Fig. 3. The TNV reconstructions corresponding to different $\lambda$ values are shown in Fig. 4. In addition, Fig. 4 shows the HAADF-STEM reconstructions, which demonstrate little difference when different $\lambda$ values are applied.

The correlation coefficient for TNV reaches the maximum when $\lambda$ equals 0.06 . Compared to the TNV reconstruction with a smaller $\lambda$, the reconstruction is less noisy and shows fewer staircase effects. For a larger $\lambda$, the image starts to show blurred edges as a result of over-regularization. These effects are similar for the TV regularization. Therefore, it is reasonable to take the reconstruction corresponding to the maximal correlation coefficients as the optimal reconstruction for the regularization method.

Fig. 5 compares the SIRT reconstruction, the TNV reconstruction and the TV reconstruction corresponding to the maximal correlation coefficients. The region-of-interest images show that the TNV reconstruction has more effective noise suppression effects and less staircase effects compared to the TV reconstruction. Both 


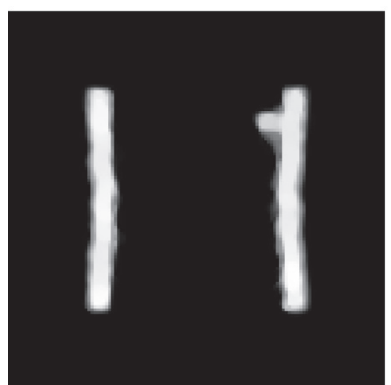

(a) TNV, 30 tilts

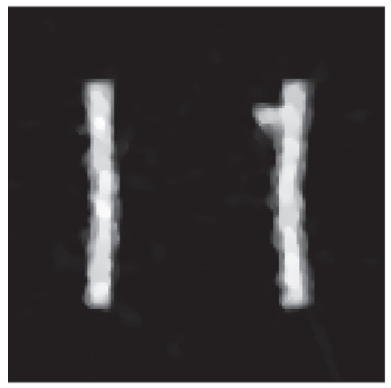

(d) $\mathrm{TV}, 30$ tilts

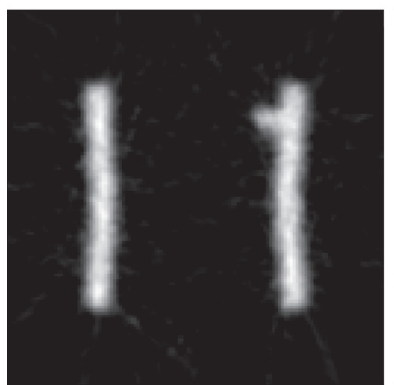

(g) SIRT, 30 tilts

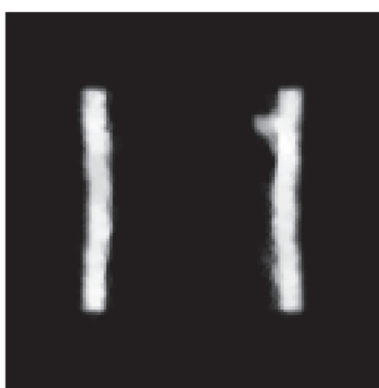

(b) TNV, 8 tilts

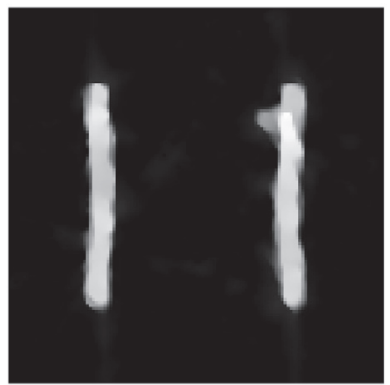

(e) TV, 8 tilts

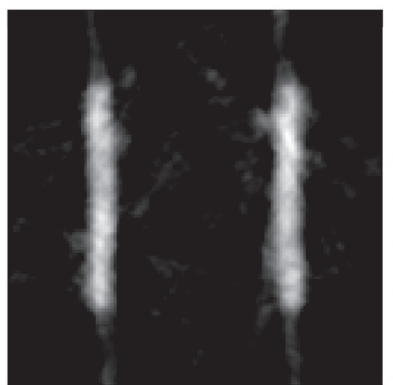

(h) SIRT, 8 tilts

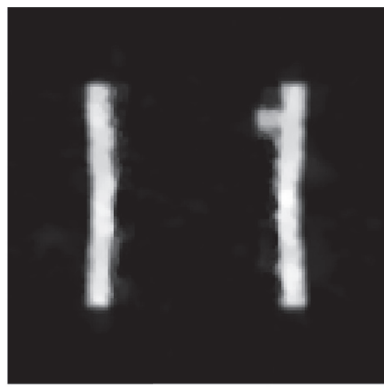

(c) TNV, 30 tilts, $1 / 3$ photon counts

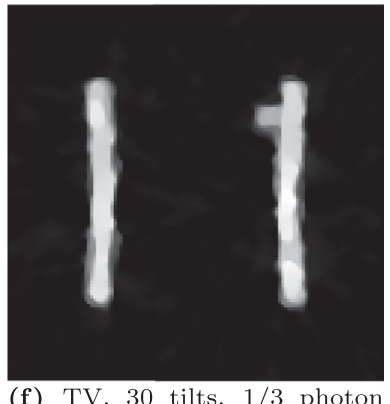

counts

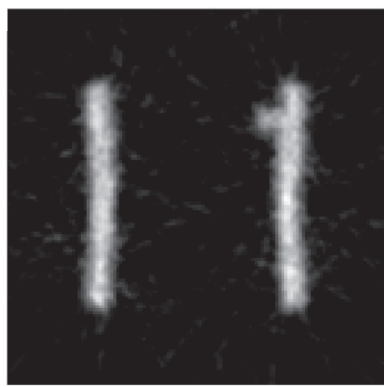

(i) SIRT, 30 tilts, $1 / 3$ photon counts

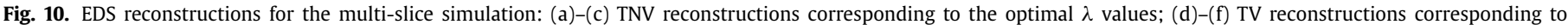
the optimal $\lambda$ values; (g)-(i) SIRT reconstructions. The EDS data in the left, middle and right columns are respectively the fully-sampled, limited-tilt and low-SNR.

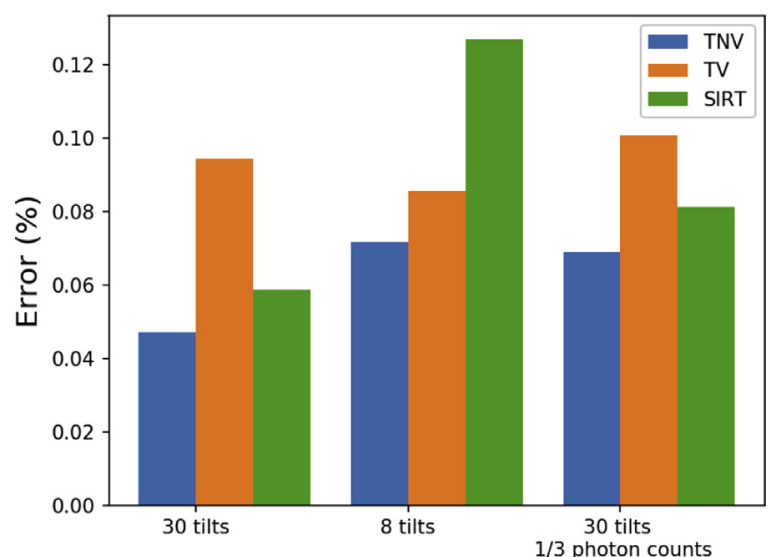

Fig. 11. Segmentation errors for the reconstructions in Fig. 10.

regularization methods reduce the missing wedge artifacts in the horizontal direction, while the TNV regularization is more effective and accurate. This is due to the augmentation by the more accurate reduction for missing wedge artifacts in the HAADF-STEM reconstruction from a large number of tilts. In addition, note that the TNV reconstruction does not show false shadows of the Ag structure from the HAADF-STEM reconstruction. Fig. 6 shows the segmented images and the segmentation errors. The plot indicates that the TNV reconstruction is more consistent with the groundtruth phantom.

\subsection{Multi-slice simulation study}

\subsubsection{Data simulation and pre-processing}

This study is based on a multi-slice simulation dataset of a $25 \times 25 \times 25 \mathrm{~nm}$ semiconductor described in [21]. Compared to the phantom simulation, the multi-slice simulation is more realistic since it includes modeling of the real physics and is based on a specimen model at the atomic level. The multi-slice simulation was performed for a high-tension of $200 \mathrm{keV}$, a convergence angle of $10 \mathrm{mrad}$ and a focused beam on the uppermost point of the specimen. The HAADF detector has an inner angle of $90 \mathrm{mrad}$ and an outer angle of $230 \mathrm{mrad}$. The focused electron probe was sampled by a $256 \times 256$ pixel array over $9.3 \times 9.3$. The EDS elemental maps were generated by summing the probability of characteristic emission.

The model is a region of a PMOS finFET. It consists of several layers placed on top of a $\mathrm{Si}$ region sequentially: $\mathrm{O}, \mathrm{HfO}_{2}$, Ta, and $\mathrm{TiAlN}_{2}$. Between the $\mathrm{HfO}_{2}$ layer and the Ta layer there is a car- 
bon nanoparticle contaminant. There are pinholes in the $\mathrm{HfO}_{2}$ layer which are filled with Ta. We focus on the EDS reconstruction for $\mathrm{Ta}$. The distribution of amorphous Ta is homogeneous in the specimen, for which the total-variation regularization can be applied. Also, the HAADF-STEM data have a large Z-contrast for Ta compared to the other elements. Therefore, the HAADF-STEM reconstruction can be used to augment the EDS reconstruction.

The raw dataset is noise-free and is at atomic resolution $(\approx$ $0.049 \mathrm{~nm} / \mathrm{pixel}$ ), where atomic-scale structures are clearly present. When we tried to add strong noise to the images, the atomic-scale structures were strongly affected by the noise. Therefore, to make the data more suitable for studying noisy data, we down-sampled the simulation data to a lower resolution. Hence, the simulated images were binned from $512 \times 512$ pixels to $128 \times 128$ pixels. After that, the images were filtered by a Gaussian filter $(\sigma=1.0$ pixel) to simulate the effect of a less tightly focused beam. Fig. 7(a) and (b) show two examples of the HAADF-STEM projection images. The process of binning approximated a low-resolution simulation. In real experiments, for lower resolution a less tightly focused beam should be used so that the entire area after binning pixel will be filled by the beam. In the end, we selected the tilt series of HAADF-STEM images from $2^{\circ}$ to $178^{\circ}$ every $2^{\circ}$, while the tilts at zone-axis $\left(0^{\circ}\right.$ and $\left.90^{\circ}\right)$ were excluded due to the channeling effects.

Similar to the phantom simulation, we generated the maps corrupted by Poisson noise from the noiseless simulation data. In this simulation, we focus on comparing the reconstructions made from different cases of data. Thus we made three data cases for different EDS datasets combined with the same HAADF-STEM data. In the first case, the fully sampled EDS data consists of 30 tilts from $2^{\circ}$ to $176^{\circ}$ for every $6^{\circ}$, of which two examples are shown in Fig. 7 (c) and (d). The average image intensity is about 16.4. Both the second and third cases simulate the scenarios when the total electron dose is reduced by a factor of 3 . The second one is a limited-tilt case, where the number of tilts is decreased from 30 to 8 . The angular range is from $2^{\circ}$ to $170^{\circ}$ for every $24^{\circ}$. The third case is a low-SNR case. The number of tilts is again 30 , while the noiseless maps were scaled by a factor of 3 to simulate reducing the dose. In this case, the average image intensity is about 5.8. For this case, two examples are given in Fig. 7(e) and (f). To all the maps, a Gaussian filter ( $\sigma=0.8$ pixel) was applied as a denoising preprocessing step.

Fig. 8(a) and Fig. 8(b) respectively show the SIRT reconstructions from the noiseless HAADF-STEM and EDS data, for the 2D slice indicated by the dashed line in Fig. 7. This EDS reconstruction is regarded as the ground-truth. In addition, Fig. 8(c) shows the ground-truth segmented image for the EDS reconstruction. Note that there is a 'tip' feature on the top right of Ta reconstruction, which is a simulated semiconductor defect.

\subsubsection{Reconstruction results}

Similar to the phantom study, we performed reconstructions with different regularization parameters and calculated the correlation coefficients w.r.t. the ground-truth, which are plotted in Fig 9. Fig. 10 shows the TNV and TV reconstructions corresponding to the maximal correlation coefficient values as well as the SIRT reconstructions. We segmented these reconstructions using the thresholding values corresponding to minimal segmentation error. Fig. 11 shows the segmentation errors.

In the fully-sampled case, for which the data quality is relatively good, the maximal correlation coefficients are close for each method. The optimal TNV reconstruction image is less noisy than the TV reconstruction. The SIRT reconstruction is smooth while showing less sharper edges. Thus, although the SIRT reconstruction has slightly higher correlation coefficient, the segmentation error is

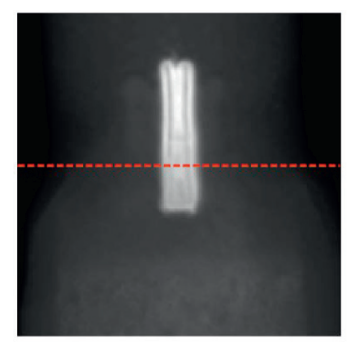

(a) HAADF-STEM image at $0^{\circ}$

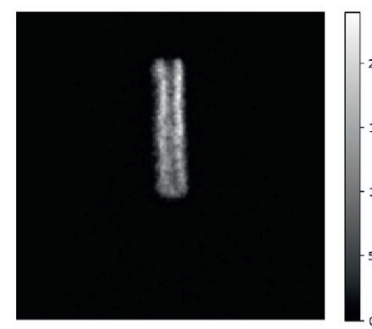

(c) EDS elemental map for $\mathrm{Ti}$ at $0^{\circ}$

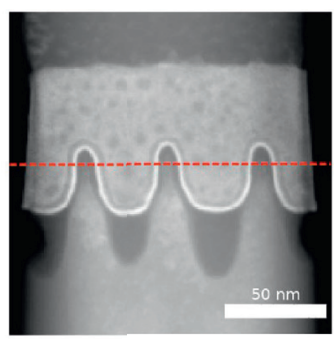

(b) HAADF-STEM image at $90^{\circ}$

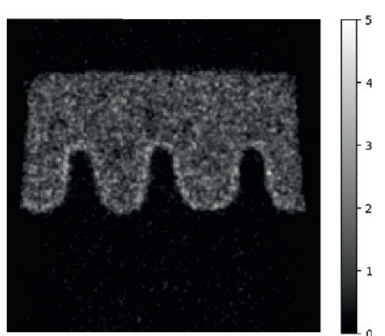

(d) EDS elemental map for $\mathrm{Ti}$ at $90^{\circ}$
Fig. 12. Examples of tilt images for the semiconductor sample. The elemental maps shown here are after Gaussian smoothing. The red dashed lines indicate the position of the reconstructed slice. The colorbars indicate the image intensities corresponding to the X-ray counts. The tilt axis is vertical. (For interpretation of the references to colour in this figure legend, the reader is referred to the web version of this article.)

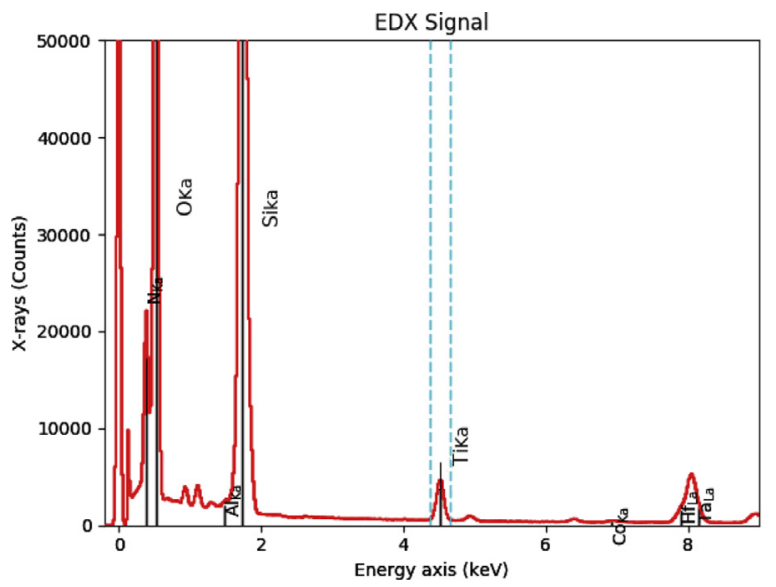

Fig. 13. An example of the X-ray spectrum for total counts integrated over the spectrum image at $0^{\circ}$. The $\mathrm{Y}$ axis corresponds to the total number of $\mathrm{X}$-ray counts in the spectrum image at each energy channel. The black lines indicate the character-

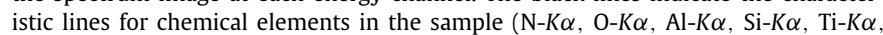
$\mathrm{Co}-K \alpha$, Hf- $L \alpha$, Ta- $L \alpha)$. The dashed lines indicate the integration windows $(0.25 \mathrm{keV})$ for extracting the Ti-K $\alpha$ elemental maps at $4.51 \mathrm{keV}$.

larger than the TNV reconstruction as the edge information is less accurate.

For the limited-tilt case, the maximal correlation coefficient for TNV reconstruction is larger than the coefficient for TV reconstruction, which is in turn larger than the coefficient for SIRT reconstruction. The SIRT reconstruction loses the 'tip' defect feature and corresponds to a large segmentation error, while the TV reconstruction is still affected by staircase effects and has rounded ends. In contrast, the TNV reconstructions show edges that are more accurate and overlap with the HAADF-STEM reconstruction. Also, the 'tip' feature is clearer. For the low-SNR case, the SIRT and TV reconstructions have more accurate structure compared to the limited-tilt case, however, are also more noisy. In comparison, the TNV reconstruction is both smooth and has accurate structure. 


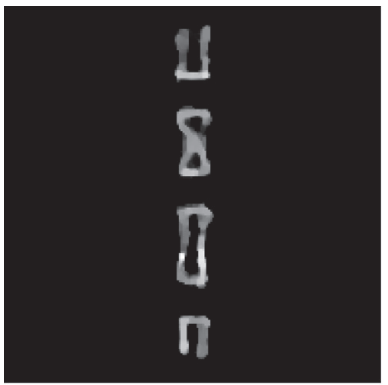

(a) TNV, 47 tilts

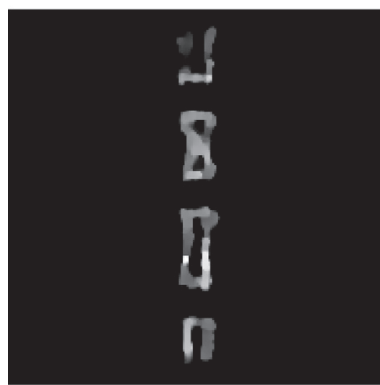

(d) $\mathrm{TV}, 47$ tilts

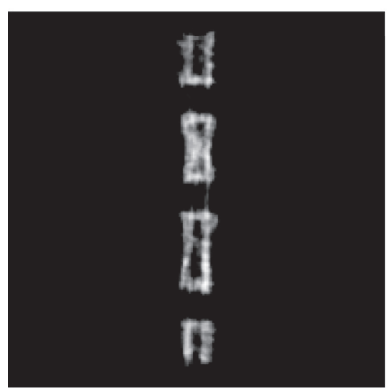

(g) SIRT, 47 tilts

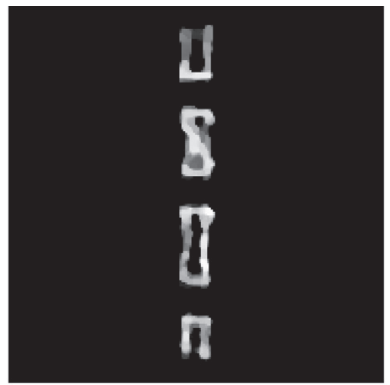

(b) TNV, 26 tilts

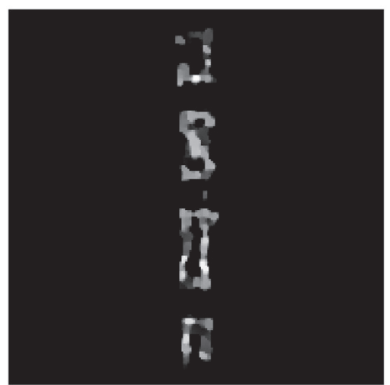

(e) TV, 26 tilts

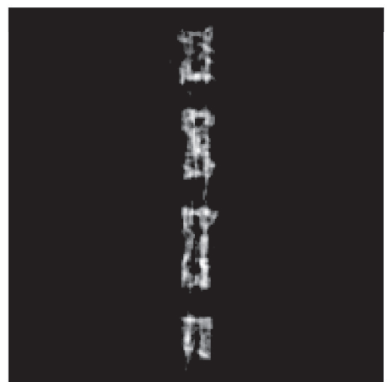

(h) SIRT, 26 tilts

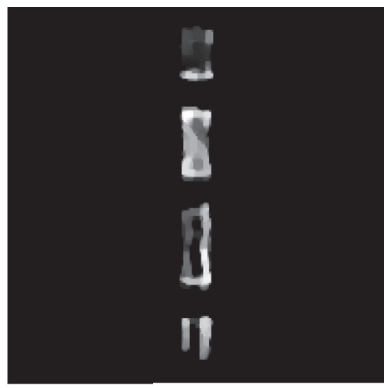

(c) TNV, 14 tilts

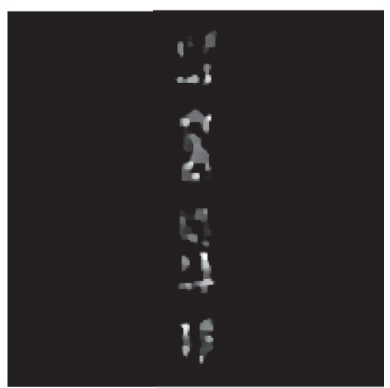

(f) $\mathrm{TV}, 14$ tilts

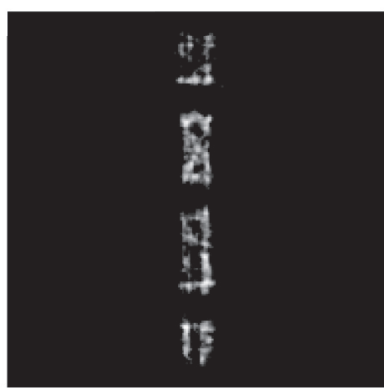

(i) SIRT: 14 tilts

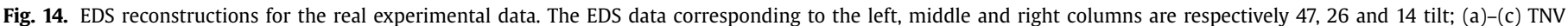
reconstructions; (d)-(f) TV reconstructions; (g)-(i) SIRT reconstructions.

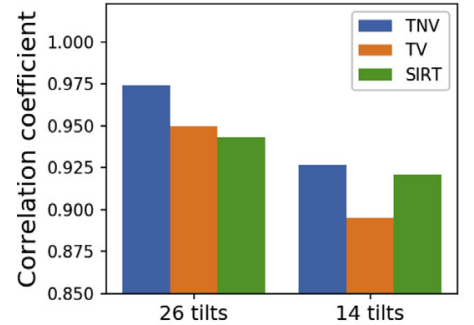

(a) Correlation coefficients

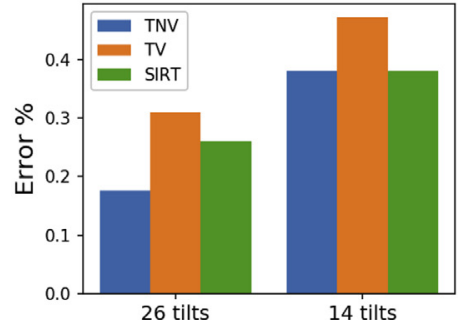

(b) Segmentation errors

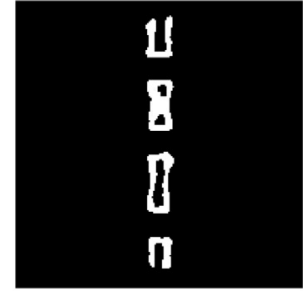

(c) Binary image used to compute the segmentation error.

Fig. 15. Measurements for the 26-tilt and 14-tilt EDS reconstructions in Fig. 14.

In general, the TNV reconstruction method outperforms the TV method and SIRT when the number of tilts is reduced or the data SNR is reduced. Comparing the TNV reconstruction image for the limited-tilt case and the low-SNR case, we notice that the latter one is slightly better than the former one. In particular, the 'tip' feature is more accurately reconstructed in the latter one. However, we should not draw a general conclusion based on this, as the result may vary depending on the structure or noise levels of reconstructions.

\subsection{Real experiment}

\subsubsection{Data acquisition and preparation}

In the last part of this section, we apply the TNV-regularized reconstruction method to real experimental data. The sample is a pillar-shape semiconductor consisting of 8 chemical elements $(\mathrm{N}$, $\mathrm{O}, \mathrm{Al}, \mathrm{Si}, \mathrm{Ti}, \mathrm{Co}, \mathrm{Hf}$ and Ta) [22]. The data were acquired using a FEI Titan electron microscope equiped with the SUPER-X system with 4 SDD X-ray detectors. The sample was placed on a Fishione on- 
axis rotation tomography holder, which allows $360^{\circ}$ rotation and avoids detector shadowing [23]. For HAADF-STEM, a tilt series of

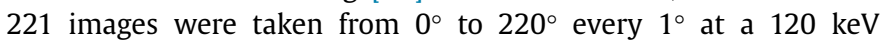
high-tension. The angular range was chosen as the maximal angular range allowed by the sample holder in a single smooth acquisition sequence. Two projection images at orthogonal angles are shown in Fig. 12(a) and (b). For EDS, a tilt series of 47 spectrumimage data-cubes were acquired for an angular range from $0^{\circ}$ to $216^{\circ}$ at approximately every $5^{\circ}$. The convergence angle was 10 mrad, the high-tension was $120 \mathrm{keV}$ and the probe current was 280 pA. The data acquisition time was about 270 seconds per tilt, which corresponds to a dwell time of $4.11 \mathrm{~ms} /$ pixel for the image of $256 \times 256$ pixels. The images were later cropped to $192 \times 192$ pixels. In addition, another tilt series of HAADF-STEM projection images aligned to the EDS measurement were taken simultaneously.

Fig. 13 plots the spectrum of total X-ray counts integrated over the spectrum images at $0^{\circ}$, where characteristic lines of all the presenting elements are indicated, among which we focus on the chemical element $\mathrm{Ti}$. In this case, $\mathrm{Ti}$ is suitable for applying the TNV-regularized reconstruction method. It has a relatively low Zcontrast compared to the heavy element Ta that surrounds it. This property can be utilized to augment the EDS reconstruction. Fig. 12(c) and (d) shows two elemental maps of Ti. The elemental maps were extracted by integrating the spectrum images at the $\mathrm{Ti}-K \alpha$ line $(4.51 \mathrm{keV})$ for an integration window of $0.25 \mathrm{keV}$. Neither background subtraction nor PCA denoising has been applied. The maps were then smoothed using a Gaussian filter for $\sigma=0.8$ pixel.

For the alignment between tilt images, the tilt series of HAADFSTEM images were aligned using the cross-correlation approach implemented in the FEI Inspect3D software. After that, the HAADFSTEM images co-acquired with EDS were aligned to the images at the same tilt in the large HAADF tilt series. Lastly, the same alignment settings were applied to the Ti elemental maps.

\subsubsection{Reconstructions and results}

In addition to the EDS data of 47 tilts, we also removed some tilts to investigate the TNV-regularized reconstruction method for 26 and 14 tilts respectively. Note that in this case, as there is no ground truth measurement, we cannot compute the variation of correlation coefficients w.r.t. the parameters like in the simulation studies. Instead, we first performed reconstructions for different $\lambda$ values from the 47-tilt data, and chose one that shows the strongest noise-suppression and no over-regularization effect. For TNV and TV, we selected the reconstructions for $\lambda=0.04$ and $\lambda=0.10$ respectively. After that, we applied the same regularization parameters to the 26-tilt and 12-tilt datasets. The reconstructions are shown in Fig. 14.

For the 47-tilt reconstructions, the TNV and TV reconstruction are similar to each other. Although for both the noise is suppressed and sharp edges are enhanced, the edges in the TNV reconstruction are slightly less noisy compared to the TV reconstruction. This is more obvious for the 26-tilt case when the TV reconstruction shows strong staircase-like patterns, while the TNV reconstruction is still smooth. For the 12 tilt case, the TV reconstruction and SIRT reconstruction are very noisy, while the TNV reconstruction is smooth and still shows structural information similar to the 47tilt reconstruction.

Fig. 15(a) shows the correlation coefficients of the 26-tilt and 14-tilt reconstructions w.r.t. the 47-tilt reconstructions corresponding to the same method. For the 26-tilt case, the correlation coefficients show that the TNV reconstructions are more linearly related to the 47-tilt reconstruction, which indicates that this reconstruction is more accurate even when some EDS data is missing. Fig. 15(b) shows the segmentation errors w.r.t. the segmented image in Fig. 15(c), which was segmented based on the reconstruc- tion image Fig. 14(a). For the 26-tilt case, quantitative information based on the TNV reconstruction is more accurate compared to the others. Nevertheless, for the 14-tilt case, although the correlation coefficient and segmentation error for the TNV reconstruction are slightly smaller than those for the TV reconstructions, they are nearly the same as for the SIRT reconstruction. This may be because the image is so blurred that the metrics fail.

\section{Discussion and conclusion}

In this paper, a new tomographic reconstruction approach based on EDS is proposed. The EDS reconstruction is performed together with a HAADF-STEM reconstruction, and they are jointly regularized by a shared TNV term. Using TNV regularization, it is possible to suppress noise and accurately reconstruct from a limited number of tilts, similar to TV regularization. TNV regularization further incorporates that the reconstruction images have sparse gradients that point in the same directions. Therefore, it encourages the EDS reconstruction to form edges overlapping with the more accurate and less noisy HAADF-STEM reconstruction.

The proposed method has been investigated on a phantom simulation, a multi-slice simulation and a real experimental dataset. For all the datasets, we used a large number of high-quality HAADF-STEM tilt images to augment the EDS reconstruction from only a small number of tilts. Note that even if the number of HAADF-STEM tilt images is smaller, the EDS reconstruction should still be augmented by the method due to the high SNR of HAADFSTEM. Also note that it is still an unsolved question how to set the regularization parameter automatically.

For the phantom simulation, the TNV method demonstrates noise suppression effects and correction of missing wedge artifacts. The reconstructed edges match those of the HAADF-STEM reconstruction. In addition, the reconstructed image shows no false structures resembling the structures in the HAADF-STEM reconstruction. For the multi-slice simulation, we compared the reconstructions for reduced dose. The reconstructions regularized by TNV are consistently more accurate compared to those regularized by TV or without regularization. Lastly, the TNV regularization was applied to real experimental data, and also shows effective noise suppression and sharp-edge enhancement. The TNV reconstruction made from 26 tilts still shows structures similar to the many-tilt reconstruction, while quantitative characterization may fail if the number of tilts is decreased to 14 .

As an alternative to the method proposed here, the TNV regularization could also be incorporated in a sequential way: first perform the HAADF-STEM reconstruction, then use the reconstruction as a TNV prior to perform the EDS reconstruction. The sequential approach is more computationally efficient if the same HAADF-STEM reconstruction can be repeatedly used. Also, it gives the opportunity to perform the HAADF-STEM reconstruction using different reconstruction methods with various priors. However, these need further research and experimental validation and will be studied in the future. Also, we will explore to combine multiple elements together with one TNV term.

In conclusion, the proposed method can be used to obtain more accurate reconstructions for data acquired using the conventional data acquisition scheme or for existing datasets. Particularly, it can be used to augment the noisy EDS reconstruction for elements of low concentrations, which may be most interesting to characterize. Moreover, using the proposed method, it is possible to adopt shorter data acquisition time or smaller beam currents while keeping nearly the same image quality. It also paves the way for developing a faster EDS tomography pipeline when the next-generation EDS detectors with higher detection rates are available. 


\section{Acknowledgement}

This work is part of the research programme "Automated multimodal tomography for sub-22nm IC nodes" with project number 13314 which is partly financed by the Netherlands Organisation for Scientific Research (NWO). It is also supported by Swedish Foundation of Strategic Research Industrial PhD grant ID14-0055 and Elekta. The authors would like to thank Dr. Richard Aveyard for providing the multi-slice simulation data and useful discussions, and Dr. Yang Qiu (imec, Leuven, Belgium - presently at South University of Science and Technology of China) and Dr. Hugo Bender (imec, Leuven, Belgium) for the experimental data set of the semiconductor pillar sample and discussion on the reconstruction results.

\section{References}

[1] M. Watanabe, D.B. Williams, The quantitative analysis of thin specimens: a review of progress from the Cliff-Lorimer to the new $\zeta$-factor methods, J. Microsc. 221 (2) (2006) 89-109.

[2] K. Lepinay, F. Lorut, R. Pantel, T. Epicier, Chemical 3D tomography of $28 \mathrm{~nm}$ high $\mathrm{K}$ metal gate transistor: STEM XEDS experimental method and results, Micron 47 (2013) 43-49.

[3] M. Burger, S. Osher, A Guide to the TV Zoo. In: Level Set and PDE Based Reconstruction Methods in Imaging. Lecture Notes in Mathematics, vol 2090, Springer, Cham, pp. 1-70.

[4] E.Y. Sidky, C.-M. Kao, X. Pan, Accurate image reconstruction from few-views and limited-angle data in divergent-beam CT, J. X-ray Sci. Tech. 14 (2006) 119-139.

[5] S.J. LaRoque, E.Y. Sidky, X. Pan, Accurate image reconstruction from few-view and limited-angle data in diffraction tomography, J. Opt. Soc. Am. A 25 (7) (2008) 1772-1782.

[6] B. Goris, W. Van den Broek, K.J. Batenburg, H. Heidari Mezerji, S. Bals, Electron tomography based on a total variation minimization reconstruction technique, Ultramicroscopy 113 (2012) 120-130.

[7] P. Burdet, Z. Saghi, A.N. Filippin, A. Borrás, P.A. Midgley, A novel 3D absorption correction method for quantitative EDX-STEM tomography, Ultramicroscopy 160 (2016) 118-129.
[8] D. Zanaga, T. Altantzis, L. Polavarapu, L.M. Liz-Marzn, B. Freitag, S. Bals, A new method for quantitative XEDS tomography of complex heteronanostructures, Particle Particle Syst. Characterization 33 (7) (2016) 396-403.

[9] D.S. Rigie, P.J.L. Rivișre, Joint reconstruction of multi-channel, spectral CT data via constrained total nuclear variation minimization, Phys. Med. Biol. 60 (5) (2015) 1741.

[10] K.M. Holt, Total nuclear variation and Jacobian extensions of total variation for vector fields, IEEE Trans. Image Process. 23 (9) (2014) 3975-3989.

[11] J. Duran, M. Moeller, C. Sbert, D. Cremers, Collaborative total variation: a general framework for vectorial TV models, SIAM J. Imaging Sci. 9 (1) (2016) 116-151.

[12] P.A. Midgley, M. Weyland, 3D Electron microscopy in the physical sciences: the development of Z-contrast and EFTEM tomography, Ultramicroscopy 96 (3) (2003) 413-431.

[13] P.A. Midgley, M. Weyland, J.M. Thomas, B.F.G. Johnson, Z-Contrast Tomography: a technique in three-dimensional nanostructural analysis based on rutherford scattering, Chem. Commun. (2001) 907-908.

[14] Z. Zhong, B. Goris, R. Schoenmakers, S. Bals, K.J. Batenburg, A bimodal tomographic reconstruction technique combining EDS-STEM and HAADF-STEM, Ultramicroscopy 174 (2017) 35-45.

[15] A.C. Kak, M. Slaney, Principles of Computerized Tomographic Imaging, IEEE Press, 1988

[16] K.E. MacArthur, M. Heggen, R.E. Dunin-Borkowski, Differentiating the structure of PtNi octahedral nanoparticles through combined ADFEDX simulations, Adv. Struct. Chem. Imaging 4 (1) (2018) 2.

[17] Z. Chen, M. Weyland, X. Sang, W. Xu, J. Dycus, J. LeBeau, A. D’Alfonso, L. Allen, S. Findlay, Quantitative atomic resolution elemental mapping via absolute-scale energy dispersive X-ray spectroscopy, Ultramicroscopy 168 (2016) 7-16.

[18] J. Gregor, T. Benson, Computational analysis and improvement of SIRT, IEEE Trans. Med. Imaging 27 (7) (2008) 918-924.

[19] W.J. Palenstijn, K.J. Batenburg, J. Sijbers, The ASTRA tomography toolbox, in: 13th International Conference on Computational and Mathematical Methods in Science and Engineering, 2013, pp. 1-7.

[20] J. Adler, H. Kohr, O. Öktem, Operator discretization library, 2017.

[21] R. Aveyard, B. Rieger, Tilt series STEM simulation of a $25 \times 25 \times 25 \mathrm{~nm}$ semiconductor with characteristic X-ray emission, Ultramicroscopy 171 (2016) 96-103.

[22] Y. Qiu, P.V. Marcke, O. Richard, H. Bender, W. Vandervorst, 3D imaging of Si FinfET devices by combined HAADF-STEM and EDS tomography, Microscopy of Semiconductor Materials, Cambridge UK, 2015.

[23] T.J. Slater, A. Janssen, P.H. Camargo, M.G. Burke, N.J. Zaluzec, S.J. Haigh, STEM-EDX tomography of bimetallic nanoparticles: a methodological investigation, Ultramicroscopy 162 (2016) 61-73. 